\title{
Structural Insights into Higher Order Assembly and Function of the Bacterial Microcompartment Protein PduA*
}

Received for publication, March 31, 2014, and in revised form, May 1, 2014 Published, JBC Papers in Press, May 29, 2014, DOI 10.1074/jbc.M114.569285

Allan Pang ${ }^{\ddagger 1,2}$, Stefanie Frank ${ }^{\S 1}$, lan Brown ${ }^{\S}$, Martin J. Warren ${ }^{\S 3}$, and Richard W. Pickersgill ${ }^{\ddagger 4}$

From the ${ }^{\ddagger}$ School of Biological and Chemical Sciences, Queen Mary University of London, Mile End Road, London E1 4NS, United Kingdom and the S School of Biosciences, University of Kent, Canterbury, Kent CT2 7NJ, United Kingdom

Background: PduA is a major shell protein in 1,2-propanediol utilization microcompartments.

Results: The profound influence of Lys-26 and Arg-79 on hexagonal tiling is revealed both in the crystal and in vivo.

Conclusion: Interactions seen in the crystal are important for sheet and nanotube assembly in the bacterial cytoplasm.

Significance: This work provides knowledge for the engineering of PduA for stability and porosity for synthetic biology applications.

Bacterial microcompartments are large proteinaceous assemblies that are found in the cytoplasm of some bacteria. These structures consist of proteins constituting a shell that houses a number of enzymes involved in specific metabolic processes. The 1,2-propanediol-utilizing microcompartment is assembled from seven different types of shell proteins, one of which is PduA. It is one of the more abundant components of the shell and intriguingly can form nanotubule-like structures when expressed on its own in the cytoplasm of Escherichia coli. We propose a model that accounts for the size and appearance of these PduA structures and underpin our model using a combinatorial approach. Making strategic mutations at Lys-26, Val51, and Arg-79, we targeted residues predicted to be important for PduA assembly. We present the effect of the amino acid residue substitution on the phenotype of the PduA higher order assemblies (transmission electron microscopy) and the crystal structure of the K26D mutant with one glycerol molecule bound to the central pore. Our results support the view that the hexamer-hexamer interactions seen in PduA crystals persist in the cytoplasmic structures and reveal the profound influence of the two key amino acids, Lys-26 and Arg-79, on tiling, not only in the crystal lattice but also in the bacterial cytoplasm. Understanding and controlling PduA assemblies is valuable in order to inform manipulation for synthetic biology and biotechnological applications.

* This work was supported by Biotechnology and Biological Sciences Research Council Grants BB/H013180/1 and BB/I013334/1 and the Higher Education Council of England.

$\because$ Author's Choice-Final version full access.

The atomic coordinates and structure factors (codes 4P7T and 4P7V) have been deposited in the Protein Data Bank (http://wwpdb.org/).

${ }^{1}$ Both authors contributed equally to this work.

${ }^{2}$ Recipient of studentship funding from Queen Mary University of London. Present address: Dept. of Chemistry and Biochemistry, UCLA, Los Angeles, CA 90095.

${ }^{3}$ To whom correspondence may be addressed: School of Biosciences, University of Kent, Canterbury, Kent CT2 7NJ, United Kingdom. Tel.: 44-1227823743; E-mail: m.j.warren@kent.ac.uk.

${ }^{4}$ To whom correspondence may be addressed: School of Biological and Chemical Sciences, Queen Mary University of London, Mile End Road, London E1 4NS, United Kingdom. Tel.: 44-2078828444; E-mail: r.w. pickersgill@qmul.ac.uk.
Bacterial microcompartments (BMCs) ${ }^{5}$ are polyhedral cellular inclusions found in several bacterial species (1-3). BMCs were discovered in cyano- and chemotrophic bacteria by transmission electron microscopy (TEM) of thin sections and were at first mistaken for phage capsids, because they share similar size and shape (4). BMCs are, in fact, composed of a protein shell encapsulating the enzymes involved in carbon fixation (carboxysomes) (5) or of a metabolic pathway (metabolosomes) $(6-10)$. The shell-forming proteins contain bacterial microcompartment domains (11). The majority of shell proteins consist of a single BMC domain (Pfam 00936) in each subunit and assemble into hexamers. Some shell proteins consist of a tandem repeat of the Pfam 00936 fold and form pseudohexameric trimers (12-14). It is plausible that the vertices are occupied by pentameric shell proteins (Pfam 03319).

The shell of the 1,2-propanediol utilization (Pdu) metabolosome is composed of seven shell proteins: PduA, PduB, PduJ, PduK, PduN, PduT, and PduU $(1,15,16)$. Previous work on the synthesis of empty Pdu microcompartments showed that not all of these seven $p d u$ genes encoding shell proteins are necessary for the formation of heterologous microcompartments in Escherichia coli (17). PduA, PduB, PduB', PduJ, PduK, and PduN were identified as the minimum shell components to form a non-aberrant empty Pdu microcompartment. Among the shell proteins, PduA is a major shell component of the Pdu metabolosome shell (18). PduA has been shown to interact with the majority of the other shell proteins and, thus, could potentially act as a scaffold for the assembly of the microcompartment (17). Deletion of PduA from a construct harboring the minimum number of genes for the formation of empty BMCs resulted in the formation of elongated filamentous structures that no longer resembled bacterial microcompartments (17). Interestingly, when PduA is overproduced alone in E. coli, it is able to form regular nanotube-like structures within the cytoplasm of the cell. A serendipitously obtained PduA construct $\left(\mathrm{PduA}^{*}\right)$ with an extra 23 residues at the $\mathrm{C}$ terminus of the protein resulted in a more soluble protein and greater density of the nanostructures, giving greater visual impact in micrographs

\footnotetext{
${ }^{5}$ The abbreviations used are: $\mathrm{BMC}$, bacterial microcompartment; TEM, transmission electron microscopy; Pdu, 1,2-propanediol utilization.
} 


\section{Assembly and Function of PduA}
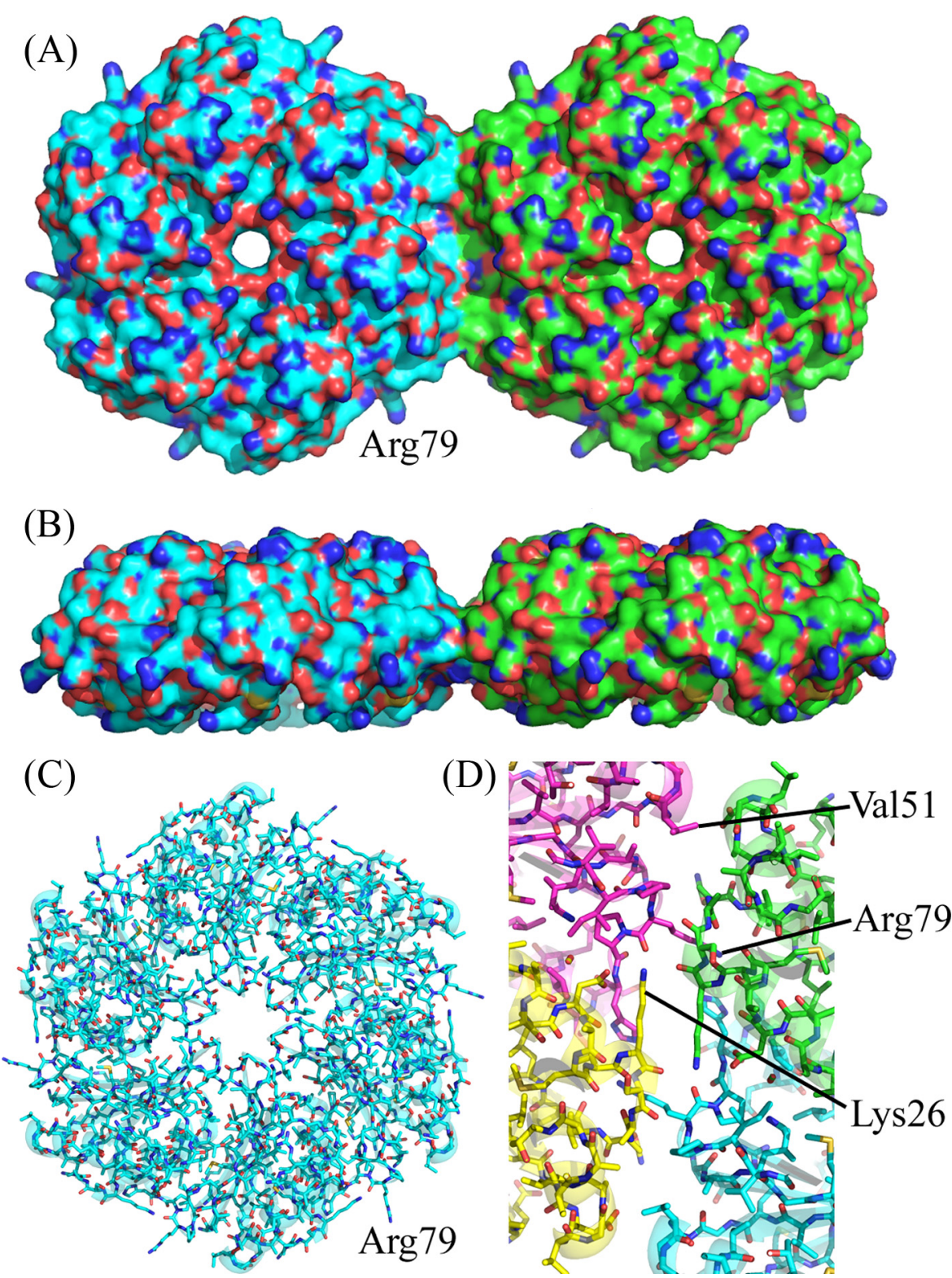

FIGURE 1. Crystal structure of native PduA (Protein Data Bank accession code 3NGK) (19). Six bacterial microcompartment protein PduA subunits form a hexamer with strict 6-fold rotational symmetry, and adjacent hexamers tile a surface. $A$, a surface representation of two PduA hexamers, one with cyan carbon atoms and one with green. One hexamer-hexamer interface is represented; this interaction is repeated to form a sheet of molecules. Arginine 79 can be seen protruding from each subunit of the hexamer for which adjacent hexamers are not drawn. $B$, the same two hexamers as in $a$ but now rotated about the $x$ axis by $90^{\circ}$ to illustrate the narrow strip of the edge of the hexamer involved in the hexamer-hexamer interaction. C, stick representation of a single PduA hexamer; the three residues chosen for mutation to probe hexamer-hexamer interactions can be clearly seen: Lys-26, Val-51, and Arg-79. D, subunit colored representation of the hexamer-hexamer interaction surface showing how one edge of the hexamer is constructed of two adjacent PduA subunits. Approximately 1,200 $\AA^{2}$ are buried at the interface between two adjacent hexamers. Arg-76 and Lys-26 contribute most to the buried (accessible) surface area, contributing 140 and $77 \AA^{2}$, respectively. Val-51 contributes a more modest but not insignificant $57 \AA^{2}$.

(17). In longitudinal sections through E. coli cells producing PduA*, thin parallel filamentous structures were observed, whereas in cross-sections, honeycomb-like structures were observed (17).

The crystal structure of PduA from Salmonella enterica (Protein Data Bank accession code 3NGK) (19) revealed that the PduA subunits pack closely together to form a biologically authentic hexamer. Of the $\sim 20,400-\AA^{2}$ total surface of the isolated PduA subunit, $\sim 9,600 \AA^{2}$ are buried in the hexamer. The native PduA hexamers in the crystal have strict 6-fold rotational symmetry (Fig. 1) and tile within the crystal lattice (of space group P622) with adjacent hexamers separated by $67.2 \AA$ (the $a$ axis of the cell). Four PduA subunits come together to form the edge between two hexamers, and together they bury only $\sim 1,200 \AA^{2}$ (Fig. $1 D$ ). It was suggested that although this is a narrow edge (Fig. $1 B$ ), the tiling of hexamers is probably closely similar to that occurring on a facet of the microcompartment (17).

Here, we suggest a model for the filamentous nanostructure produced by PduA* in the E. coli cytoplasm and provide mutational evidence in support of the model. We use a combination of mutagenesis, TEM, and crystallography to study morphological changes of the nanostructures resulting from changes of hexamer-hexamer interactions. We provide evidence that the 
interactions between PduA hexamers seen in the crystal persist in the bacterial cytoplasm. Moreover, we present the crystal structure of a PduA variant that has a substrate analog bound to the central pore.

\section{EXPERIMENTAL PROCEDURES}

Bioinformatics and Modeling-The interface between PduA hexamers in the crystal was analyzed using CCP4 programs PDBSET and AREAIMOL (20). PyMOL (21) was used for visualization and to produce the figures of molecules. Sequence conservation across several species of PduA was assessed using ClustalW (22). SymmDock $(23,24)$ was used to generate symmetry-related hexamers, which were then manually docked to form nanotubes using PyMOL (21).

Generation of PduA* Mutants-The sequence of Citrobacter freundii PduA is essentially the same as that from S. enterica. The only difference is that the $C$. freundii protein is one residue shorter; it lacks the C-terminal glutamine of S. enterica. The cloning of PduA* into pET3a has been described previously (17). PduA* is a more soluble form of C. freundii PduA with Arg-93 instead of Ser-93 and 23 additional C-terminal residues: LVKDPAANKARKEAELAAATAEQ. The Stratagene QuikChange protocol was used to generate five mutants, K26A, K26D, V51A, V51D, and R79A, using mutagenic primers. The correct sequence of the constructs was verified by DNA sequencing (Eurofins). PduA* K26D was subcloned into pET14b to facilitate PduA* K26D overproduction as a fusion protein with an $\mathrm{N}$-terminal $\mathrm{His}_{6}$ tag in E. coli. In this paper, PduA will be used in place of the PduA* designation.

Transmission Electron Microscopy - E. coli BL21(DE3) pLysS cells were transformed with PduA and the five PduA mutant constructs. Bacteria cells were grown in $50 \mathrm{ml}$ of lysogeny broth containing $100 \mathrm{mg} /$ liter ampicillin with aeration at $37^{\circ} \mathrm{C}$. Upon reaching an $A_{600}$ of 0.8 , protein production was induced with $0.4 \mathrm{~mm}$ isopropyl $\beta$-D-thiogalactoside, and the cultures were incubated by shaking overnight at $18{ }^{\circ} \mathrm{C}$. Harvested cells were resuspended in $2 \mathrm{ml}$ of fixative consisting of $2.5 \%$ glutaraldehyde in phosphate-buffered saline (PBS). The cells were pelleted and washed twice with PBS to remove traces of the fixing solution. Cells were then stained for $1 \mathrm{~h}$ in $1 \%$ osmium tetroxide and washed with PBS before dehydration. Dehydration was carried out by placing the samples into a solvent gradient: $60 \%$ industrial methylated spirit overnight, 90\% industrial methylated spirit for $15 \mathrm{~min}, 100 \%$ industrial methylated spirit for 15 min, and $100 \%$ dried ethanol twice for $2 \mathrm{~h}$. The cells were embedded by first incubating them overnight in 30\% agar low viscosity resin in dried ethanol and then embedding them for $180 \mathrm{~min}$ in $100 \%$ agar low viscosity resin that was constituted for a block with medium hardness (three changes of resin). The samples were placed in $0.5-\mathrm{ml}$ embedding tubes, centrifuged for $5 \mathrm{~min}$ at $4,000 \times g$ to concentrate the cells to the tip of the tube, and incubated at $60{ }^{\circ} \mathrm{C}$ overnight to polymerize. Specimens were thin sectioned with a diamond knife on an RMC MT-6000-XL ultramicrotome, collected on copper grids, and post-stained with $5 \%$ uranyl acetate for $30 \mathrm{~min}$ at $60{ }^{\circ} \mathrm{C}$ and $0.1 \%$ lead citrate for $10 \mathrm{~min}$ at room temperature. Sections were then observed and photographed with a JEOL-1230 transmission electron microscope.
Production, Purification, and Crystallization of PduA K26DBL21*(DE3) pLysS harboring pET14b-PduA K26D were cultured for $21 \mathrm{~h}$ at $28{ }^{\circ} \mathrm{C}$ in 1 liter of $2 \times$ YT medium supplemented with $100 \mathrm{mg} /$ liter ampicillin and $35 \mathrm{mg} /$ liter chloramphenicol. Cells were harvested by centrifugation $(10 \mathrm{~min}$, $4,000 \times g$ ) and resuspended in a total volume of $30 \mathrm{ml}$ of binding buffer (50 mм Tris- $\mathrm{HCl}, \mathrm{pH}$ 8.0, $0.5 \mathrm{~m} \mathrm{NaCl}, 10 \mathrm{~mm}$ imidazole). Cells were lysed by sonication, and cell debris was removed by centrifugation $(35,000 \times g$ for $20 \mathrm{~min})$. The recombinant protein was purified using IMAC. The bound fraction was washed with increasing amounts of imidazole and eluted in $400 \mathrm{~mm}$ imidazole. The histidine tag was cleaved off of the purified protein by overnight incubation with thrombin. PduA K26D was further purified, and thrombin was removed by passing it over a size exclusion column (Superdex 200 Global 10/30) equilibrated with 50 mm Tris-HCl, pH 8.0, 100 mm NaCl. PduA K26D crystals were grown by hanging drop vapor diffusion. Type I crystals were harvested from protein drops $(2.9 \mathrm{mg} / \mathrm{ml})$ equilibrated against a reservoir of $1.3 \mathrm{M}$ sodium citrate tribasic dihydrate, $0.1 \mathrm{~m}$ sodium HEPES, pH 7.9. The reservoir for type II crystals was $1.0 \mathrm{M}$ sodium citrate tribasic dihydrate and $0.1 \mathrm{M}$ Tris at $\mathrm{pH} 8.5$, and the protein used was at $6.3 \mathrm{mg} / \mathrm{ml}$. Reservoir augmented with $15 \%$ glycerol was used as a cryoprotectant, and x-ray diffraction data to 1.72 and $1.93 \AA$ resolution were collected from the two different crystal forms at the Diamond Light Source (I03) using the PILATUS $6 \mathrm{M}-\mathrm{F}$ pixel detector. Data were processed using XDS (25) and XDSME and scaled using SCALA (26). The reduced data were analyzed using MOLREP (27), REFMAC (28), and COOT (29), and the quality of the final model was assessed using PROCHECK (30).

\section{RESULTS}

Modeling of Higher Order PduA Structures Observed in TEMWe have cultured, embedded, and analyzed an E. coli strain overproducing PduA using transmission electron microscopy. In agreement with previous observations (17), we observed that PduA gives rise to higher order structures that resemble honeycombs in transverse sections (Fig. 2a) and tubes in longitudinal sections (Fig. 2b). Measurement of the structures formed by PduA reveals that the structures are $20.4 \pm 1.1 \mathrm{~nm}$ in diameter (20 measurements) and as long as the bacterial cell (1-2 $\mu \mathrm{m})$. The honeycomb appearance in cross-section is suggestive of a bundle of tubular structures with a tendency to close hexagonal packing (Fig. 2a). We suggest that these filamentous structures are nanotubes. The lack of electron density in the lumen of the nanotubes is consistent with this hypothesis (it should be noted that a combination of positive (osmium) and negative (uranyl) staining has occurred during sample preparation). When viewed in longitudinal section, the tubes appear as pairs of lines corresponding to the negatively stained walls of the tubes (Fig. $2 b$ ). We propose that in the cytoplasm, the PduA molecules will tend to form a hexagonally tiled sheet similar to that seen in the crystal lattice for native protein (17). This sheet could form a tube by rolling up so that the outward facing vertex of one edge of the sheet could bind to the inward facing vertex of the other edge of the sheet ( $A$ to $A$ in Fig. $3 A$ ). The need to interdigitate the vertices constrains the number of hexamers per turn to be 


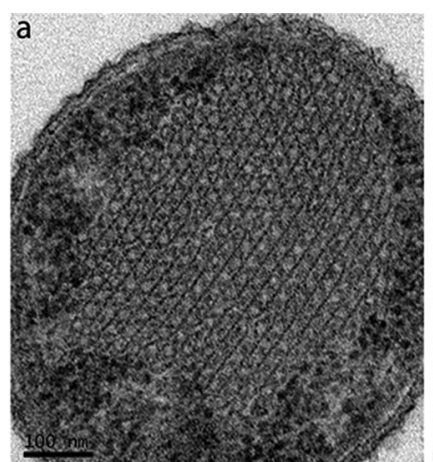

b
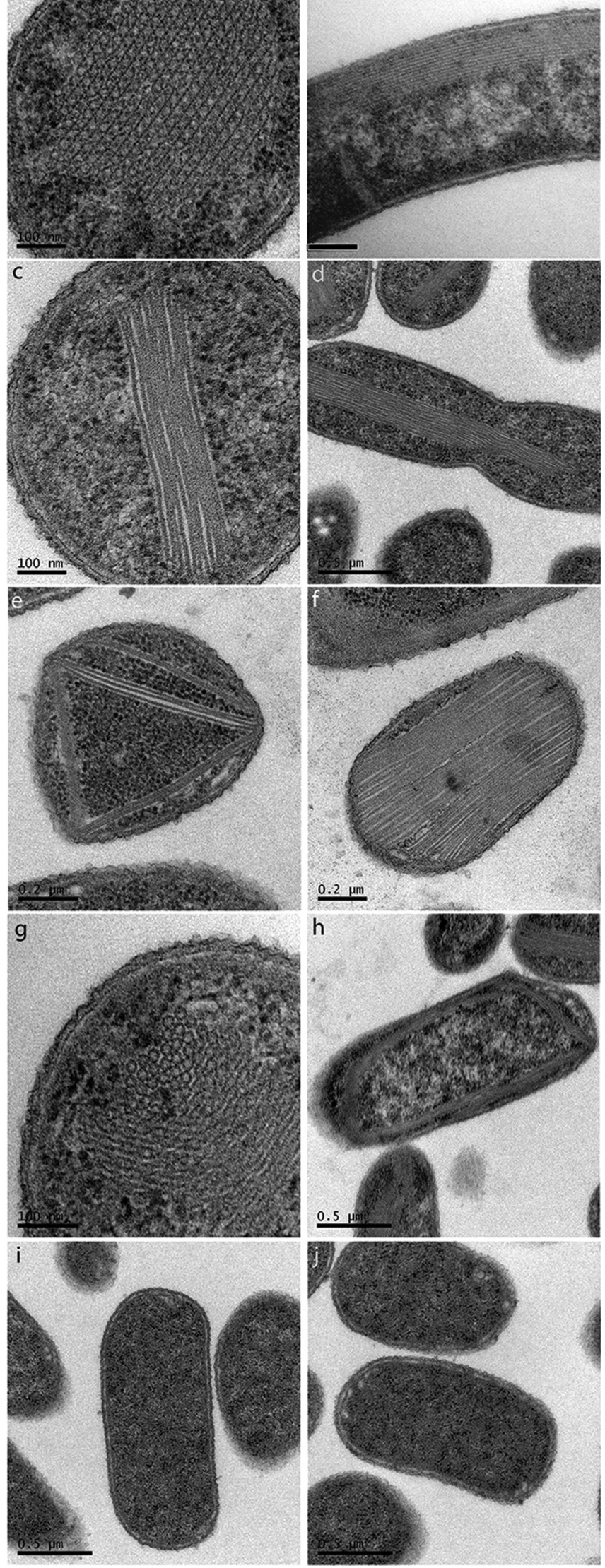

(A)

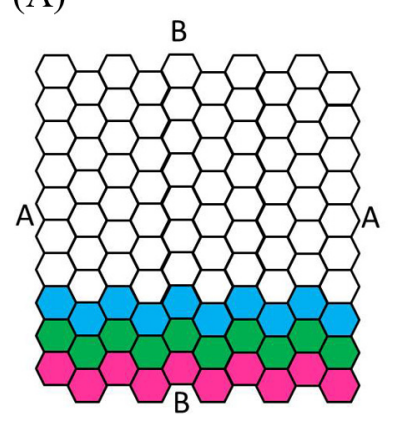

(B)

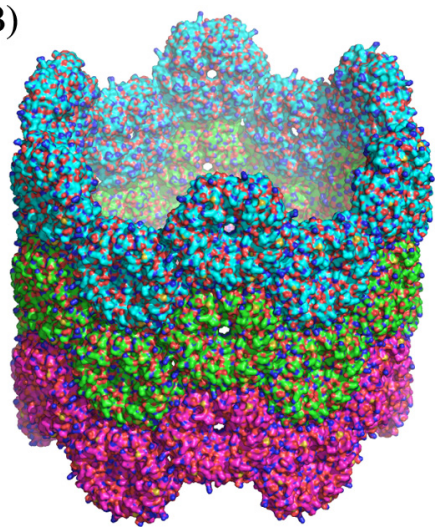

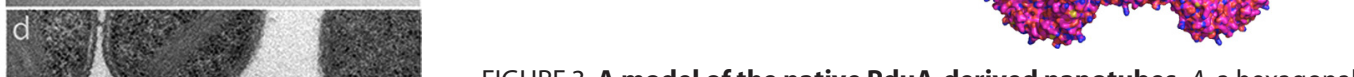

FIGURE 3. A model of the native PduA-derived nanotubes. $A$, a hexagonal sheet of PduA molecules can be rolled into a tube in one of two ways; either $A$ can be connected to $A$, or $B$ can be connected to $B$. In the model shown in $B$, the sheet is rolled so that $A$ and $A$ are brought together, and there are 12 hexamers per ring of the protein nanotube. The packing of hexamers within the ring and between rings is equivalent.

even. Alternatively, the edges of the hexagons could meet ( $B$ to $B$ in Fig. $3 A$ ).

Model building studies with the edges of the PduA hexamers perpendicular to the tube axis ( $A$ to $A$ in Fig. $3 A$ ) revealed that to produce a tube consistent with the experimentally determined diameter of $20 \mathrm{~nm}$ required 12 hexamers (72 subunits) per turn (Fig. 3B). Because the hexamers are very stable, and the hexamer-hexamer junctions are far less so, we argue that it is the angle between adjacent hexamers that will change to accommodate the curvature to bring the first and 12th hexamers together. In fact, 12 hexamers per turn require an internal angle of $150^{\circ}$; the required curvature is therefore achieved by tilting succeeding hexamers through $30^{\circ}$. This is surprisingly readily achieved because the hexamers are wedge-shaped when viewed perpendicular to the 6-fold axis (Fig. $1 B$ ). In fact, the key interaction between the hexamers involving lysine 26 is maintained when adjacent hexamers are placed together at a $30^{\circ}$ angle. No distortion of the hexamer is needed; nor are there any interpenetrating surfaces. The alternative way of rolling up the sheet, with hexamer edges approximately parallel to the tube axis and preserving the antiparallel hydrogen bonding of adjacent lysine 26 residues, produces a helix of 10 PduA hexamers per turn, of 20-nm diameter and with a pitch of two hexamers (138 $\AA$ ). This structure can therefore be described as a two-start helix, each of pitch $138 \AA$. The precise arrangement of the hexamers within the nanotube remains to be resolved, but the

$\overline{\text { FIGURE 2. Transmission electron microscopy of native PduA ( } a \text { and } b \text { ) and }}$ hexamer-hexamer interaction mutants $(c-j)$ produced in $E$. coli cells. Images on the left show transverse sections, and images on the right show longitudinal sections through the cell (except for the last row, where both are longitudinal sections). $a$ and $b$, native PduA forms higher order structures that resemble honeycombs in transverse section $(a)$ and tube-like structures in longitudinal sections (b). $c-h$, phenotypes of strains producing $\mathrm{K} 26 \mathrm{~A}$ ( $c$ and $d$ ), R79A ( $e$ and $f$ ), and V51 A ( $g$ and $h$ ) interaction mutants. Both the K26A and the R79A mutant PduA micrographs show sheetlike assemblies that are layered. There is no evidence of PduA nanotubes for these mutants in transverse sections ( $c$ and $e$ ). The R79A PduA structures are more regularly packed than K26A PduA-derived structures. V51A PduA is the only mutant with different phenotypes in transverse $(g)$ and longitudinal $(h)$ view. V51A forms protein nanotubes of $18.3 \pm 1.3-\mathrm{nm}$ diameter (e), similar to the native PduA. No structures were observed for the K26D (i) or V51D (j) mutations. Scale bar in b, 0.5 $\mu \mathrm{m}$; other scale bars can be seen more clearly in the images. 


\section{Assembly and Function of PduA}

PduA
PduB_79-161
PduB_185-267
PduJ
PduK_1-96
PduU_45-116
PduT_3-76
PduT_95-173
PduA
PduB_79-161
PduB_185-267
PduJ
PduK_1-96
PduU_45-116
PduT_3-76
PduT_95-173

MQQEALGMVET-KGLTAAIEAADAMVKSANVMLVGYEKI-GSGLVT-----VIVRG-DVG 52 ---RS IGILGARTGAGPHIMAADEAVKATNTEVVS IELPRDTKGGAGHGSLIILGGNDVS 57 ---RACGVIVG-APASVGVLMADTALKSANVEVVAYSSP-AHGTSFSNEAILVISG-DSG 54 -MNNALGLVET-KGLVGAIEAADAMVKSANVOLVGYEKI-GSGLIT-----VMVRG-DVG 51 -MKQSLGLLEV-SGLALAISCADVMAKAASITLVGLEKTNGSGWMV-----IKIIG-DVA 52 ----AIGILTI-TPSEASIIACDIATKSGAVEIGFLDRF--TGAVV-------LTG-DVS 45 ---QAIGILEL-TSIAKGMEAGDAMLKSANVNLL-VSKTICPGKEL-----LMLGG-DVG 49 ---QAVGIVET-WSVAACICAADRAVKASNVTLVRVHMAFGIGGKCY----MVVAG-DVS 51 : * : : $\quad$ : * * : :

AVKAATDAGAAAARNV-GEVKAVHVIPRPHTDV--EKILPKGIS- 93 DVKRGIEVALKELDRTFGDVYANEAG------------------ 83

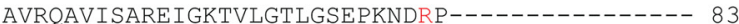
AVKAAVDAGSAAASAV-GEVKSCHVIPRPHSDV--EAILPKSA-- 91 SVQAAISTGVSFADQR-DGLVAHKVISRPGDGILSHSVTPESESE 96 AVEYALKQVTRTLGEM-MRETACPITRT---------------- 72 AVQQAIATGTSLAG---DMLVDSLVLPNIH-------------- 76 DVNNAVTVASESAGEK-GLLVYRSVIPRP---------------- 79 *. .

FIGURE 4. Sequence alignment of shell proteins from C. freundii (PduA, PduB, PduJ, PduK, PduU, and PduT) using ClustalW2. PduB and PduT were aligned using their individual domains determined by InterProScan4; where a subset of residues was used, the range is indicated. Residue conservation is indicated by an asterisk, and similarity is indicated by a colon or period. Residues selected for mutation are in red.

important characteristics of both models are that the interhexamer interactions are preserved, the bending of adjacent hexamers is readily accommodated, and the concave surface of the hexamer faces outward and the convex surface faces the lumen of the tube so that the outside surface resembles the texture of a golf ball.

Analysis of the Native PduA Hexamer-Hexamer InterfaceTo probe our tube model, we aimed to disrupt the hexagonal tiling of PduA seen in the native crystal lattice and explore the effect this has on the formation of higher order structures visible in the bacterial cell cytoplasm. Calculation of changes in solvent accessibility when native PduA hexamers are brought together to form a sheet of molecules reveals residues that are at the interface (data not shown, but readily calculated using methods under "Experimental Procedures"). Lys-26 and Arg-79 have the highest solvent accessibility changes (77 and $139 \AA^{2}$, respectively) and would therefore be expected to be of greatest importance for tiling in crystals and in microcompartment facets. Arg-79 can be clearly seen protruding from the isolated hexamer in Fig. $1 C$; it is positioned to plug into the adjacent hexamer in the sheet. Val-51 (57- $\AA^{2}$ solvent accessibility change) also occurs at the hexamer-hexamer interface, and its mutation was thought to influence the stability of hexamer-hexamer interactions although not as profoundly as Lys-26 and Arg-79. Substitutions planned were either replacements by alanine (truncation of the side chain) or by aspartate, which would be most perturbing to the interface when the substitution is close to the 2 -fold axis between hexamers, bringing the substituted residues close together. Lys-26 to Asp was therefore anticipated to be most disruptive because the aspartates introduced would be proximal in the absence of structural rearrangement (Fig. 1D), whereas the substitution of Val-51 for Asp was anticipated to be least disruptive because this residue is further from the 2-fold axis relating adjacent hexamers, and therefore aspartate and its symmetry mate would not be in close proximity (Fig. 1D). Lysine 26 is involved in a characteristic antiparallel interaction, a hydrogen bond, with the 2-fold related hexamer (side-chain NZ to main-chain carbonyl; Fig. $1 D$ ) and is conserved across the shell proteins (Fig. 4).
Transmission Electron Microscopy Observations of the PduA Mutants-To study the effect the amino acid substitutions have on the phenotype of the tube-like structures, E. coli strains producing the Lys-26, Val-51, and Arg-79 mutants were cultured, embedded, sectioned, and visualized under the transmission electron microscope. Micrographs of strains producing the alanine mutants K26A and R79A show higher order structures, which appear to be multiple parallel sheets of proteins (Fig. 2, $c-f)$. We suggest that these sheets are assembled from PduA hexamers in the bacterial cytoplasm. Multiple units of R79Aderived sheets were observed in a single cell, giving rise to striking arrangements that span the cell and even seem to stretch cells, causing shape changes (Fig. 2e). The V51A mutant is the only variant that forms structures with a distinct appearance in longitudinal and transverse (Fig. 2, $g$ and $h$ ) section. In crosssection, PduA V51A appears to form honeycomb-like structures of $18.3 \pm 1.3 \mathrm{~nm}$ in diameter (40 measurements). They are less regularly packed but of similar diameter (within error) to the structures seen previously with native PduA $(20.4 \pm 1.1$ $\mathrm{nm})$. Overexpression of K26D or V51D mutant PduAs resulted in no higher order structures being observed by TEM in the bacterial cytoplasm (Fig. 2, $i$ and $j$ ) despite the proteins being produced in quantities suitable for crystallization and structure determination (see results below).

Crystal Structures of the PduA K26D Mutant-The lysine 26 mutant is the most important to study structurally because Lys-26 is conserved in all shell proteins (Fig. 4) and contributes significantly to the interface between 2 -fold related hexamers in the native crystals. Two crystal forms were obtained for this mutant using commercially available screens. There is clear electron density for residues 4-89 in nine copies of the PduA subunit across the two crystal forms, and the C-terminal residues, including the additional 23 residues, are not seen in the electron density maps. The fewest residues are seen for subunit $C$ in type I crystals, where only residues 6-79 are clearly defined. In type I crystals, the K26D PduA hexamers form long strips of molecules in the P2 lattice (Table 1) (i.e. the interaction shown in Fig. 5A) are repeated in one direction only (onedimensional tiling in the diagonal of the $a c$ plane) with a hex- 
TABLE 1

Crystallographic data and refinement statistics for the K26D PduA mutant structure

\begin{tabular}{|c|c|c|}
\hline Crystal & Type I & Type II \\
\hline Space group & $\mathrm{P} 2_{1}$ & $\mathrm{P} 2_{1}$ \\
\hline $\begin{array}{l}\text { Subunits in asymmetric } \\
\text { unit }\end{array}$ & 6 & 6 \\
\hline Cell parameters $(\AA)$ & $\begin{array}{c}45.2,93.3,63.1 \\
\beta=105.0^{\circ}\end{array}$ & $\begin{array}{c}68.0,53.3,68.1 \\
\beta=117.5^{\circ}\end{array}$ \\
\hline Resolution range $(\AA)$ & $60.89-1.72(1.76-1.72)^{a}$ & $60.35-1.93(2.0-1.93)$ \\
\hline Observed reflections & $269,196(18,852)$ & $75,170(5,639)$ \\
\hline No. of unique reflections & $53,279(3,903)$ & $29,930(2,254)$ \\
\hline Completeness (\%) & $99.4(98.6)$ & $94.3(95.5)$ \\
\hline Multiplicity & $5.1(4.8)$ & $2.5(2.5)$ \\
\hline$\langle I / \sigma(I)\rangle$ & $13.9(2.2)$ & $10.7(2.4)$ \\
\hline$R_{\text {merge }}(\%)^{b}$ & $0.06(0.617)$ & $0.05(0.363)$ \\
\hline$R$-work/R-free & $0.183 / 0.230$ & $0.198 / 0.257$ \\
\hline $\begin{array}{l}\mathrm{RMSD}^{c} \text { (bonds) } \\
(\AA) / \text { RMSD angle } \\
\text { (degrees) }\end{array}$ & $0.026 / 2.507$ & $0.016 / 1.750$ \\
\hline Wilson $B$-factor $\left(\AA^{2}\right)$ & 29.1 & 32.9 \\
\hline No. of protein atoms & 3,520 & 3,636 \\
\hline No. of water molecules & 318 & $180 / 1$ glycerol \\
\hline $\begin{array}{l}\text { Ramachandran plot } \\
\text { statistics: residues in } \\
\text { most favored/additional } \\
\text { regions }(\%)\end{array}$ & $98 / 2$ & $97 / 3$ \\
\hline \multicolumn{3}{|c|}{$\begin{array}{l}{ }^{a} \text { The highest resolution range and parameters for that range are presented in } \\
\text { parentheses. } \\
{ }_{b} R_{\text {merge }}=\Sigma_{h k l} \Sigma_{i}\left|I_{i}(h k l)-(I(h k l))\right| / \Sigma_{h k l} \Sigma i I_{i}(h k l) \text {, where } I_{i}(h k l) \text { is the } i \text { th obser- } \\
\text { vation of reflection } h k l \text {, and }\langle I(h k l)\rangle \text { is the weighted average intensity for all ob- } \\
\text { servations of reflection } h k l \text {. }\end{array}$} \\
\hline
\end{tabular}

amer separation in the tiling distance of $67.4 \AA$, just slightly greater than the native distance of $67.2 \AA$. The strips of K26D PduA hexamers then pack alternately back to face to form the three-dimensional crystal lattice. The formation of strips is understandable when the conformation of Arg-79 is considered. Along the axis forming the strip, Arg-79 interacts with a 2-fold related hexamer (Fig. 5B) to form intermolecular hydrogen bonds; Arg-79 NE hydrogen-bonds to Asp-26', and Arg-79 $\mathrm{NH} 1$ and NH2 hydrogen-bond the main-chain carbonyl of Arg$79^{\prime}$, but this interaction is not preserved at all potential hexamer-hexamer interfaces. This difference in conformation is most pronounced for the adjacent interface, where Arg-79 is involved in intramolecular interactions (C-subunit mentioned earlier). Here, Arg-79 hydrogen-bonds to Asp-22 and mainchain carbonyls of residues 83 and 84 (Fig. 5C).

In contrast, in the type II P2 lattice, the K26D PduA hexamers do tile (two-dimensional tiling), but the separation of hexamers is greater than seen for native PduA at 68.0 and $68.1 \AA$ along $a$ and $c$, respectively, compared with $67.2 \AA$ for native PduA, resulting in an interface that is clearly not as tight (Fig. $5 A$ ) as in the native PduA sheets (Fig. 1A). Remarkably, Arg-79 is now interacting with its 2-fold related symmetry mate in an elongated conformation reminiscent of the lysine interaction seen in native PduA (Fig. 5D). Here, the guanidinium group of Arg-79 is hydrogen-bonding to Asp-26' (Arg-79 NE), Asp-22' (Arg-79 $\mathrm{NH}_{2}$ ), and the carbonyl-oxygen atoms of Asp-22' and Arg-79' (the prime indicates a 2-fold related residue).

In both crystal forms, the regularity of the tiling is lost in that the K26D PduA hexamer no longer has strict 6-fold symmetry, and the separation between hexamers is greater than in native PduA sheets $(67.2 \AA)$. In the first form, strips of hexamers are formed, and in the second, sheets with rather poor hexamerhexamer interactions are seen. It is perhaps not surprising then

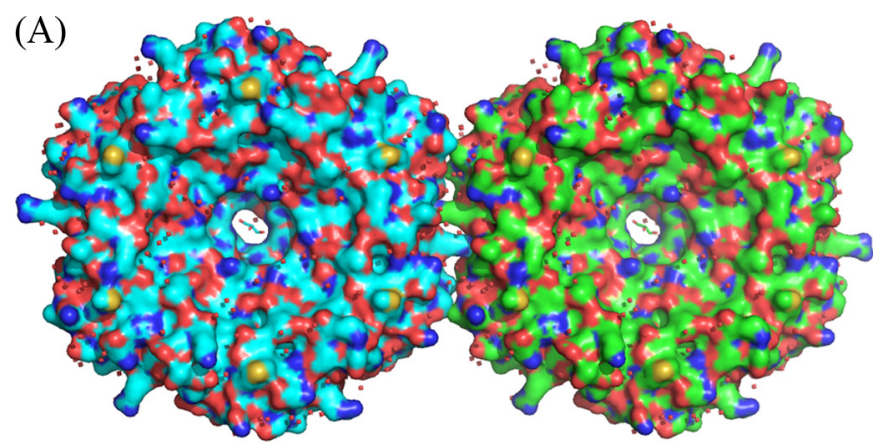

(B)

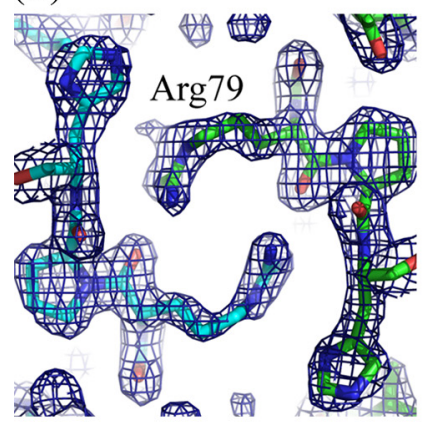

(C)

(D)
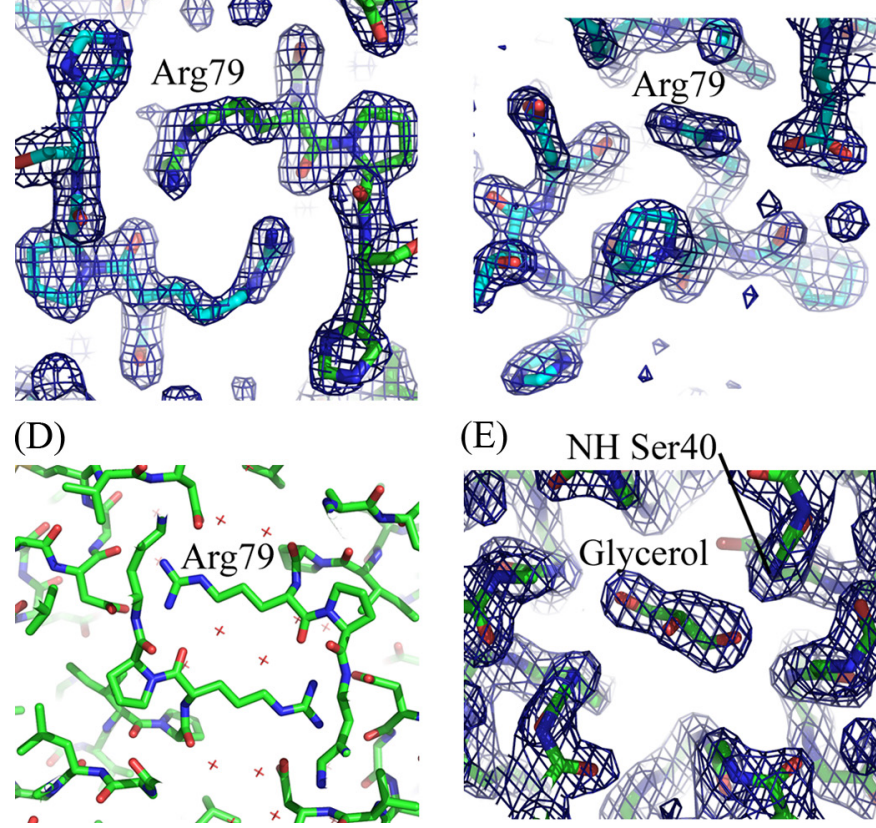

(E)

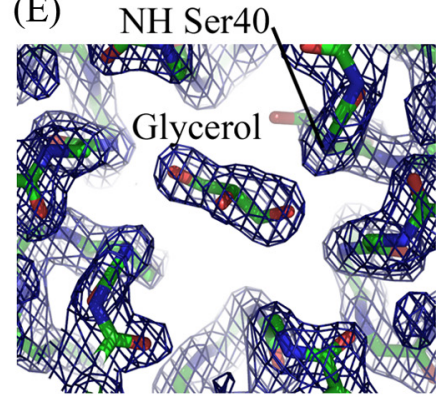

FIGURE 5. Crystal structure of the K26D PduA mutant. $A$, the strict 6-fold symmetry is lost in the K26D PduA mutant structure (type I crystals, $1.72 \AA$ resolution), as can be best illustrated by examining Arg-79, which no longer has the regularity that can be seen for native PduA in Fig. $1 C$. The interface is also more fragile. $B$, the interaction between side-by-side hexamers is now dominated by Arg-79, which forms an intermolecular hydrogen bond with the main-chain carbonyl of Arg-79 from the hexamer with which it packs, which is surprisingly reminiscent of the lysine to main-chain carbonyl seen in the native structure. Asp-26 makes intermolecular hydrogen bonds to NE of Arg-79 (not shown). C, Arg-79 in subunit F, however, is involved in intrasubunit hydrogen bonds to OE1 of conserved Asp-22 and main-chain carbonyls of residues 83 and 84 . Although adjacent hexamers can pack, as illustrated in $B$, they fail to tile in the $\mathrm{P} 2{ }_{1}$ crystal lattice. $\sigma_{\mathrm{A}}$-weighted $2 F_{\text {obs }}-F_{\text {calc }}$ electron density is shown as blue chicken wire mesh. $D$, extended antiparallel arginine residues hydrogen-bonding to both aspartates 22 and 26 as well as the carbonyl of symmetry-related Arg-79 at the hexamer-hexamer interface. $E$, a single glycerol molecule (an analog of 1,2-propanediol substrate) is seen in the central pore of the hexamer.

that no structures are seen for the K26D mutant in the bacterial cytoplasm when PduA K26D is overexpressed. A question of the effect of crystallization conditions arises because the conditions used were different from those used for crystallization of the Salmonella enterica PduA, but there is no obvious reason why the crystallization conditions would result in molecular asymmetry, whereas hexamer association is an observable cause of asymmetry. The breaking of the 6-fold symmetry allows a single glycerol molecule to be seen occupying the central pore of the hexamer (type II crystals; Fig. 5E). The O1 and O3 hydroxyls of this glycerol molecule hydrogen-bond to the 
TABLE 2

Summary of PduA hexamer interaction mutants and TEM observations

\begin{tabular}{|c|c|c|}
\hline K26 & V51 & R79 \\
\hline \multicolumn{3}{|l|}{ Characteristics } \\
\hline $\begin{array}{l}\text { Conserved across shell } \\
\text { proteins. Centrally located at } \\
\text { the interface between two } \\
\text { hexamers where it interacts } \\
\text { with its symmetry mate. }\end{array}$ & $\begin{array}{l}\text { Located towards the } \\
\text { extremity of the interface of } \\
\text { two hexamers. }\end{array}$ & $\begin{array}{l}\text { Centrally located at interface } \\
\text { between two hexamers. } \\
\text { Close to Asp83 and main- } \\
\text { chain carbonyl of Vall } 25 \text {. }\end{array}$ \\
\hline \multicolumn{3}{|l|}{ Predicted role } \\
\hline $\begin{array}{l}\text { Predicted to have a profound } \\
\text { influence on tiling through } \\
\text { anti-parallel interaction (NZ } \\
\text { to main-chain carbonyl O) } \\
\text { with two-fold related lysine } \\
26 \text {. Hexamer formation } \\
\text { results in } 77 \AA^{2} \text { becoming } \\
\text { buried. }\end{array}$ & $\begin{array}{l}\text { Less pronounced effect on } \\
\text { tiling compared to Lys } 26 \text { as } \\
\text { Val51 is further from } 2 \text {-fold } \\
\text { axis where it interacts with } \\
\text { the hydrophobic part of } \\
\text { Gly52 and Ala53. Hexamer } \\
\text { formation results in } 56 \AA^{2} \\
\text { becoming buried. }\end{array}$ & $\begin{array}{l}\text { Important for tiling through } \\
\text { hydrogen bonds with two- } \\
\text { fold related hexamer. } \\
\text { Hexamer formation results in } \\
139 \AA^{2} \text { becoming buried. }\end{array}$ \\
\hline \multicolumn{3}{|l|}{ In vivo observations (TEM) } \\
\hline \multicolumn{3}{|l|}{ Ala substitution } \\
\hline $\begin{array}{l}78 \% \text { of cells* were seen to } \\
\text { contain closely packed } \\
\text { structures, similar in } \\
\text { transverse and longitudinal } \\
\text { view interpreted as sheets of } \\
\text { hexamers. }\end{array}$ & $\begin{array}{l}\text { Honeycomb-like structures in } \\
\text { transverse section and tube- } \\
\text { like structures in longitudinal } \\
\text { view (seen in } 97 \% \text { of the } \\
\text { cells studied)*. } \\
\text { Resembles native PduA and } \\
\text { interpreted as sheets of } \\
\text { hexamers rolled up into } \\
\text { tubes. }\end{array}$ & $\begin{array}{l}\text { Closely packed structures, } \\
\text { similar in transverse and } \\
\text { longitudinal view consistent } \\
\text { with sheets of hexamers } \\
\text { (seen in } 97 \% \text { of cells } \\
\text { studied)*. }\end{array}$ \\
\hline \multicolumn{3}{|l|}{ Asp substitution } \\
\hline No structures observed** & No structures observed & Not determined \\
\hline \multicolumn{3}{|c|}{$\begin{array}{l}\text { *, } 200 \text { cells were examined in detail for structures made by PduA and mutants of } \\
\text { PduA; } 100 \% \text { of PduA cells examined showed tube-like structures. The other per- } \\
\text { centages given represent a minimum because it is possible that the structures } \\
\text { were present but did not extend throughout the bacterial cell. } \\
\text { **, Two crystal forms were obtained, the regularity of the tiling is lost, and there is } \\
\text { a breaking of strict } 6 \text {-fold symmetry axis of hexamer. }\end{array}$} \\
\hline
\end{tabular}

main-chain amides of Ser-40 of subunits on opposite sides of the hexamer axis. The separation is $9.9 \AA$ along this long axis as opposed to $8.5 \AA$ for the axis at $60^{\circ}$.

\section{DISCUSSION}

We investigate the effect of mutation of three residues identified as important in the association of hexamers on nanotube formation in the bacterial cytoplasm and, for a key mutation, $\mathrm{K} 26 \mathrm{D}$, the effect also on the packing of the hexamers in the crystal lattice (Table 2). Aspartate substitutions destabilize higher order structures to the extent that none are seen in TEM. This is consistent with the hexamer-hexamer interface seen in the crystal being important in the formation of the structures seen in TEM. For one of these mutants (K26D), crystals were obtained, and these crystals reveal more fragile hexamer-hexamer tiling than in native PduA in one crystal form (type II) and only one-dimensional tiling in the other (type I). The mutation K26D has a profound influence on the conformation of the neighboring Arg-79, such that this region of the protein no longer adopts a single conformation in the crystal, resulting in breaking of the 6-fold symmetry axis of the hexamer. This results in the imperfect tiling of PduA molecules seen in the crystals and apparent lack of tiling at all in the bacterial cytoplasm. The flexibility of the main-chain conformation around Arg-79 is a result of its position after the last $\beta$-strand of the bacterial microcompartment domain in a loop before the last $\alpha$-helix, which tolerates structural change without affecting subunit folding or hexamer formation. But the influence of this residue on hexamer symmetry and tiling is profound.

The more modest alanine substitutions allow the formation of structures that appear similar in longitudinal and transverse section. We suggest that these are sheets of molecules in the bacterial cytoplasm. Only the V51A mutant gives tube-like structures resembling those formed using PduA. This suggests that the lysine 26 and arginine 79 interactions are profoundly important in maintaining the integrity of the molecular assembly as the hexamerhexamer angle is increased from $0^{\circ}$ (tiling a plane) to $30^{\circ}$ (an angle necessary to form a tube with 12 hexamers per turn), and these interactions are preserved in our model of the nanotube. Overall, the results support the view that tiling of the microcompartment facet is due to interactions seen in the native PduA crystal lattice. They also suggest a mechanism by which the edges of the microcompartment could be formed where adjacent hexamers can associate at an out-of-plane angle of up to $30^{\circ}$. We have presented a model for the molecular architecture that underpins the nanotubes seen in TEM with PduA and V51A PduA. In addition, we have observed impressive arrangements of PduA structures that distort the cell's shape and segregate regions within the cell, confining ribosomes to certain areas. It may be possible to take advantage of these properties to permit the design of specific protein scaffolds or use them for controlled exclusion of certain regions of the bacterial cell.

Our crystallization studies have allowed us to see the first substrate analog (glycerol is closely similar to 1,2-propanediol) bound to the central pore of PduA (in the K26D mutant, which lacks 6-fold symmetry in the crystal lattice of the type II crystals), supporting the view that substrate can gain access to the microcompartment via the central pore of PduA. Compared with the long subunit pore described previously for PduB, which accommodates three glycerol molecules (31), the central pore within PduA is thin and binds a single glycerol. The contacts to the three glycerol molecules in PduB mainly involve side chains; here at the central pore, the contact is with the main-chain amide of Ser-40 from two PduA subunits. The presence of glycerol within the central channel, with coordinating loops distorted from precise 6-fold symmetry, suggests that variations from strict 6-fold symmetry seen are important for the biological function of PduA. Such insights can help in the redesign of shell protein pores for the passage of different metabolites.

In summary, structural insights into PduA have revealed how knowledge of shell proteins can be used to help the construction of large semipermeable protein scaffolds that could be employed for the targeted localization of specific pathways. Moreover, the detail on substrate binding to the pores within the shell protein can be used for the redesign of substrate entry/ product release from bacterial microcompartments, which hold significant biotechnological potential for the incorporation of pathways with toxic intermediates (32).

Acknowledgment_We acknowledge use of the Diamond Light Source (Oxford, UK). 
Note Added in Proof-Bobik and Yeates have recently published a study of residues important for the assembly of the microcompartment shell (Sinha, S., Cheng, S., Sung, Y. W., McNamara, D. E., Sawaya, M. R., Yeates, T. O., and Bobik, T. A. (2014) Alanine scanning mutagenesis identifies an asparagine-arginine-lysine triad essential to the assembly of the shell of the pdu microcompartment. J. Mol. Biol. 426, $2328-2345$.

\section{REFERENCES}

1. Bobik, T. A., Havemann, G. D., Busch, R. J., Williams, D. S., and Aldrich, H. C. (1999) The propanediol utilization (pdu) operon of Salmonella enterica serovar Typhimurium LT2 includes genes necessary for formation of polyhedral organelles involved in coenzyme $B_{12}$-dependent 1 , 2-propanediol degradation. J. Bacteriol. 181, 5967-5975

2. Shively, J. M., Bradburne, C. E., Aldrich, H. C., Bobik, T. A., Mehlman, J. L., Jin, S., and Baker, S. H. (1998) Sequence homologs of the carboxysomal polypeptide CsoS1 of the thiobacilli are present in cyanobacteria and enteric bacteria that form carboxysomes: polyhedral bodies. Can. J. Bot. 76, 906-916

3. Sriramulu, D. D., Liang, M., Hernandez-Romero, D., Raux-Deery, E., Lünsdorf, H., Parsons, J. B., Warren, M. J., and Prentice, M. B. (2008) Lactobacillus reuteri DSM 20016 produces cobalamin-dependent diol dehydratase in metabolosomes and metabolizes 1,2-propanediol by disproportionation. J. Bacteriol. 190, $4559-4567$

4. Bock, E., Düvel, D. and Peters, K. R. (1974) [Characterization of a phagelike particle from cells of Nitrobacter. I. Host-particle correlation and particle isolation (author's transl)]. Arch. Microbiol. 97, 115-127

5. Kerfeld, C. A., Heinhorst, S., and Cannon, G. C. (2010) Bacterial microcompartments. Annu. Rev. Microbiol. 64, 391-408

6. Brinsmade, S. R., Paldon, T., and Escalante-Semerena, J. C. (2005) Minimal functions and physiological conditions required for growth of Salmonella enterica on ethanolamine in the absence of the metabolosome. J. Bacteriol. 187, 8039-8046

7. Jorda, J., Lopez, D., Wheatley, N. M., and Yeates, T. O. (2013) Using comparative genomics to uncover new kinds of protein-based metabolic organelles in bacteria. Protein Sci. 22, 179-195

8. Seedorf, H., Fricke, W. F., Veith, B., Brüggemann, H., Liesegang, H., Strittmatter, A., Miethke, M., Buckel, W., Hinderberger, J., Li, F., Hagemeier, C., Thauer, R. K., and Gottschalk, G. (2008) The genome of Clostridium kluyveri, a strict anaerobe with unique metabolic features. Proc. Natl. Acad. Sci. U.S.A. 105, $2128-2133$

9. Stojiljkovic, I., Bäumler, A. J. and Heffron, F. (1995) Ethanolamine utilization in Salmonella typhimurium: nucleotide sequence, protein expression, and mutational analysis of the cchA cchB eutE eut eutG eutH gene cluster. J. Bacteriol. 177, 1357-1366

10. Walter, D., Ailion, M., and Roth, J. (1997) Genetic characterization of the pdu operon: use of 1,2-propanediol in Salmonella typhimurium. J. Bacteriol. 179, 1013-1022

11. Kerfeld, C. A., Sawaya, M. R., Tanaka, S., Nguyen, C. V., Phillips, M., Beeby, M., Yeates, T. O. (2005) Protein structures forming the shell of primitive bacterial organelles. Science 309, 936-938

12. Heldt, D., Frank, S., Seyedarabi, A., Ladikis, D., Parsons, J. B., Warren, M. J., and Pickersgill, R. W. (2009) Structure of a trimeric bacterial microcompartment shell protein, EtuB, associated with ethanol utilization in Clostridium kluyveri. Biochem. J. 423, 199-207

13. Yeates, T. O., Kerfeld, C. A., Heinhorst, S., Cannon, G. C., and Shively, J. M. (2008) Protein-based organelles in bacteria: carboxysomes and related microcompartments. Nat. Rev. Microbiol. 6, 681-691
14. Pang, A., Warren, M. J., and Pickersgill, R. W. (2011) Structure of PduT, a trimeric bacterial microcompartment protein with a $4 \mathrm{Fe}-4 \mathrm{~S}$ cluster-binding site. Acta Crystallogr. D Biol. Crystallogr. 67, 91-96

15. Havemann, G. D., and Bobik, T. A. (2003) Protein content of polyhedral organelles involved in coenzyme B12-dependent degradation of 1,2-propanediol in Salmonella enterica serovar Typhimurium LT2. J. Bacteriol. 185, 5086-5095

16. Parsons, J. B., Dinesh, S. D., Deery, E., Leech, H. K., Brindley, A. A., Heldt, D., Frank, S., Smales, C. M., Lünsdorf, H., Rambach, A., Gass, M. H., Bleloch, A., McClean, K. J., Munro, A. W., Rigby, S. E., Warren, M. J., and Prentice, M. B. (2008) Biochemical and structural insights into bacterial organelle form and biogenesis. J. Biol. Chem. 283, 14366-14375

17. Parsons, J. B., Frank, S., Bhella, D., Liang, M., Prentice, M. B., Mulvihill, D. P., Warren, M. J. (2010) Synthesis of empty bacterial microcompartments, directed organelle protein incorporation, and evidence of filamentassociated organelle movement. Mol. Cell 38, 305-315

18. Cheng, S., Sinha, S., Fan, C., Liu, Y., and Bobik, T. A. (2011) Genetic analysis of the protein shell of the microcompartments involved in coenzyme B-12-dependent 1,2-propanediol degradation by Salmonella. J. Bacteriol. 193, 1385-1392

19. Crowley, C. S., Cascio, D., Sawaya, M. R., Kopstein, J. S., Bobik, T. A., and Yeates, T. O. (2010) Structural insight into the mechanisms of transport across the Salmonella enterica Pdu microcompartment shell. J. Biol. Chem. 285, 37838-37846

20. Collaborative Computational Project, Number 4 (1994) The CCP4 suite: programs for protein crystallography. Acta Crystallogr. D Biol. Crystallogr. 50, $760-763$

21. DeLano, W. L., and Lam, J. W. (2005) PyMOL: a communications tool for computational models. Abstr. Papers Am. Chem. Soc. 230, 254-COMP

22. Larkin, M. A., Blackshields, G., Brown, N. P., Chenna, R., McGettigan, P. A., McWilliam, H., Valentin, F., Wallace, I. M., Wilm, A., Lopez, R., Thompson, J. D., Gibson, T. J., and Higgins, D. G. (2007) ClustalW and ClustalX version 2.0. Bioinformatics 23, 2947-2948

23. Schneidman-Duhovny, D., Inbar, Y., Nussinov, R., and Wolfson, H. J. (2005) PatchDock and SymmDock: servers for rigid and symmetric docking. Nucleic Acids Res. 33, W363-W367

24. Schneidman-Duhovny, D., Inbar, Y., Nussinov, R., and Wolfson, H. J. (2005) Geometry-based flexible and symmetric protein docking. Proteins 60, 224-231

25. Kabsch, W., (2010) XDS. Acta Crystallogr. D Biol. Crystallogr. 66, 125-132

26. Evans, P. (2006) Scaling and assessment of data quality. Acta Crystallogr. D Biol. Crystallogr. 62, 72-82

27. Vagin, A., and Teplyakov, A. (1997) MOLREP: an automated program for molecular replacement. J. Appl. Crystallogr. 30, 1022-1025

28. Murshudov, G. N., Vagin, A. A., and Dodson, E. J. (1997) Refinement of macromolecular structures by the maximum-likelihood method. Acta Crystallogr. D Biol. Crystallogr. 53, 240-255

29. Emsley, P., Lohkamp, B., Scott, W. G., and Cowtan, K. (2010) Features and development of Coot. Acta Crystallogr. D Biol. Crystallogr. 66, 486-501

30. Laskowski, R. A., MacArthur, M. W., Moss, D. S., and Thornton, J. M. (1993) Procheck: a program to check the stereochemical quality of protein structures. J. Appl. Crystallogr. 26, 283-291

31. Pang, A., Liang, M., Prentice, M. B., and Pickersgill, R. W. (2012) Substrate channels revealed in the trimeric Lactobacillus reuteri bacterial microcompartment shell protein PduB. Acta Crystallogr. D Biol. Crystallogr. 68, $1642-1652$

32. Frank, S., Lawrence, A. D., Prentice, M. B., and Warren, M. J. (2013) Bacterial microcompartments moving into a synthetic biological world. J. Biotechnol. 163, 273-279 